terising changes in local gene expression in endothelium sampled from control coronary arteries and from advanced coronary atherosclerotic plaques.

Methods and Results A protocol was optimised to perform laser capture microdissection for isolation of endothelium in under 20 minutes, from snap-frozen porcine coronary artery cross-sections for total RNA sequencing ( $n=5$ D374Y-PCSK9 hyperlipidaemic minipigs fed on a high fat high cholesterol diet; 10 vessels). Endothelium was sampled from control left anterior descending arteries consisting of no plaque, and from overlying advanced atherosclerotic plaque in stenotic right coronary arteries. Differential gene expression and gene ontology enrichment analyses revealed the upregulation of numerous atheroprone inflammatory genes in diseased endothelium overlying atherosclerotic plaque compared to healthy endothelium (figure 1: $\mathrm{p}<0.01, \mathrm{FDR}<0.05$, fold change $>2$ ). Biological pathways related to atherosclerosis that the differentially expressed genes are most enriched in are shown (table 1: enrichment score $>3$ ).

Conclusions We report in vivo changes in gene expression in diseased endothelium overlying advanced coronary atherosclerotic plaques, with upregulation of inflammatory genes. The differentially expressed genes are enriched in processes related to atherosclerosis, suggesting the validity of this approach to study how gene expression changes during coronary atherosclerotic plaque development in vivo. Our model system allows for further studies coupling together these readouts to a fully validated $3 \mathrm{D}$ vessel reconstruction method to co-register gene expression profiles to local blood flow data, as a novel methodology for understanding the mechanisms by which local flow disturbances may affect atherogenesis. This will provide new insights into how disturbed flow and coronary atherosclerotic plaque development are causally related. Conflict of Interest None

\section{BS19 PHOSPHODIESTERASE 1 (PDE1) INHIBITION ENHANCES PROSTACYCLIN MEDIATED SIGNALLING IN PULMONARY ARTERY SMOOTH MUSCLE CELLS THROUGH A NOVEL SELECTIVE PDE1C:PROSTACYCLIN RECEPTOR INTERACTION}

${ }^{1}$ Zaher Al Bakour, ${ }^{1}$ James Hislop, ${ }^{2}$ James Guy Breitenbucher, ${ }^{1}$ Fiona Murray. ${ }^{1}$ University of Aberdeen, Aberdeen, UK; ${ }^{2}$ Convelo Therapeutics

10.1136/heartjnl-2021-BCS.217

Introduction Pulmonary arterial hypertension (PAH) is associated with increased phosphodiesterase 1C (PDE1C) expression and activity, which accounts in part, to lower cAMP accumulation and increased proliferation of pulmonary artery smooth muscle cells (PASMC) isolated from PAH-patients: PDE1C expression correlates with increased PASMC proliferation. PAH-PASMC also have decreased prostacyclin (IP) receptor expression that limits their response to IP agonists. Recently, a phosphodiesterase 1 inhibitor (16K) has been developed for central nervous system disorders and tested in preclinical studies. Using PASMC we aimed to investigate the response of $16 \mathrm{~K}$, alone and together with the prostacyclin (IP) receptor agonist selexipag, to provide evidence for the therapeutic utility of PDE1 inhibitors for PAH.

Methods Human PASMCs were cultured under normoxia and hypoxia $(1 \% \mathrm{O} 2,72 \mathrm{hr})$ to investigate PDE1C expression (Real-time PCR) and the effect of $16 \mathrm{~K}(0.01-10 \mu \mathrm{M})$ and
Selexipag $(0.001-1 \mu \mathrm{M})$ on PASMCs proliferation (MTS) and cAMP accumulation (ELISA). PDE1C, PDE1A and PDE4B cDNA (SinoBiological) and IP cDNA (cDNA.org) were used to be stably overexpressed in HEK293 (Lipofectamine 2000, ThermoFisher). Lysosomal inhibitor (chloroquine, $100 \mu \mathrm{M}$ ) and proteasomal inhibition (MG132, $10 \mu \mathrm{M}$ ) were used to assess IP receptor degradation, via fluorescence microscopy. Q5 sit-directed mutagenesis kit was used for prostacyclin receptor PDZ domain manipulation. Experiments were performed at least three times and data presented as means $\pm \mathrm{S}$. E.M and compared by ANOVA or student t-test.

Results PDE1C mRNA is increased in PASMC exposed to hypoxia (62.9 \pm 6.9-fold increase vs. normoxia, $\mathrm{p}<0.05$, $\mathrm{n}=3$ ), which correlated with increased PDE1C protein expression and activity: increased PDE1C expression correlated with lower cAMP (103.4 \pm 4.9 vs. $56.3 \pm 3.5 \mathrm{pmol} / \mathrm{million}$ cells in normoxic and hypoxic PASMC, respectively) and increased proliferation. PDE1 inhibition (16K) restored cAMP levels $(56.3 \pm 3.5$ vs. $106.2 \pm 8.8 \mathrm{pmol} /$ million cells in control and 16K-treated hypoxic PASMC, respectively) and induced PASMC relaxation and inhibited hypoxia-induced proliferation (21 $\pm 0.03 \%$ decrease, MTS assay). Selexipag-mediated cAMP accumulation and relaxation, which was blunted in hypoxic-PASMC, was restored by $16 \mathrm{~K}$ pre-treatment. In PASMC and HEK293 we found overexpression of PDE1C correlated with increased proteasomal degradation of the IP receptor, which blunted receptor mediated responses. Overexpression of other PDEs (PDE1A or 4B) were not associated with changes in IP receptor function. Manipulating PDZ domain of IP receptor, which binds PDZK1, prevented this interaction and restored the function and expression of IP receptor. $16 \mathrm{~K}$ restored the expression of the IP receptor and its agonist induced cAMP accumulation via a cAMP-PKAdependent mechanism.

Conclusions Our data show $16 \mathrm{~K}$ increases basal and selexipagmediated cAMP accumulation, relaxation and inhibition of proliferation in hypoxic-PASMC. We uncovered a novel PDZfacilitated interaction of PDE1C with the IP receptor, such that increased PDE1C associated with PAH would limit agonist-induced cAMP accumulation and relaxation by enhancing IP degradation. Together these data provide further evidence that PDE1 selective inhibitors could represent a novel PAH treatment alone and importantly enhance the response to prostacyclin agonists.

Conflict of Interest None

\section{BS20 THE OPPOSING EFFECTS OF CHRONIC INTERLEUKIN-1 $\beta$ ON TIE2:TIE1 RATIO AND ANGIOPOIETIN1 INDUCED PI3 KINASE/AKT SIGNALING IN ENDOTHELIAL CELLS}

Harprit Singh, Jay Bilimoria. De Montfort University, Leicester, UK

\subsection{6/heartjnl-2021-BCS.218}

Introduction Angiopoietin-1 (Ang-1) is a growth factor that plays a crucial role in maintaining normal vascular function. The main role of Ang-1 is to maintain endothelial survival. Ang-1 exerts its protective effect by activating Tie- 2 receptors and subsequently the Phosphatidylinositol 3-kinase (PI3Kinase)/ AKT pathway. Tie-1 regulates Ang-1 signalling with high levels of the receptor reducing Ang-1-induced Tie-2 activation. Proinflammatory cytokines including Tumor Necrotic factor (TNF- $\alpha$ ) Interleukin $1 \beta$ (IL-1 $\beta$ ) have been implicated in a 